\title{
On the structural intricacies of a metabolic precursor: Direct spectroscopic detection of water-induced conformational reshaping of mevalonolactone
}

Sérgio R. Domingos, Cristóbal Pérez, and Melanie Schnell

Citation: The Journal of Chemical Physics 147, 124310 (2017); doi: 10.1063/1.4997162

View online: https://doi.org/10.1063/1.4997162

View Table of Contents: http://aip.scitation.org/toc/jcp/147/12

Published by the American Institute of Physics

\section{Articles you may be interested in}

Structural determination and population transfer of 4-nitroanisole by broadband microwave spectroscopy and tailored microwave pulses

The Journal of Chemical Physics 147, 154306 (2017); 10.1063/1.4991902

Communication: Structural locking mediated by a water wire: A high-resolution rotational spectroscopy study on hydrated forms of a chiral biphenyl derivative

The Journal of Chemical Physics 145, 161103 (2016); 10.1063/1.4966584

Laser ablated hydantoin: A high resolution rotational study

The Journal of Chemical Physics 147, 124312 (2017); 10.1063/1.4994799

The gas-phase structure of the asymmetric, trans-dinitrogen tetroxide $\left(\mathrm{N}_{2} \mathrm{O}_{4}\right)$, formed by dimerization of nitrogen dioxide $\left(\mathrm{NO}_{2}\right)$, from rotational spectroscopy and ab initio quantum chemistry

The Journal of Chemical Physics 146, 134305 (2017); 10.1063/1.4979182

Weak hydrogen bond topology in 1,1-difluoroethane dimer: A rotational study

The Journal of Chemical Physics 147, 094301 (2017); 10.1063/1.4994865

Intramolecular hydrogen bonding in malonaldehyde and its radical analogues

The Journal of Chemical Physics 147, 124309 (2017); 10.1063/1.4996563

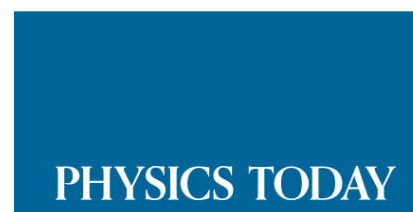

WHITEPAPERS
ADVANCED LIGHT CURE ADHESIVES

Take a closer look at what these environmentally friendly adhesive systems can do
READ NOW

PRESENTED BY

(8) MASTERBOND' 


\title{
On the structural intricacies of a metabolic precursor: Direct spectroscopic detection of water-induced conformational reshaping of mevalonolactone
}

\author{
Sérgio R. Domingos, ${ }^{1,2}$ Cristóbal Pérez, ${ }^{1,2}$ and Melanie Schnell 1,2,3,a) \\ ${ }_{1}^{1}$ Max Planck Institute for the Structure and Dynamics of Matter, Luruper Chaussee 149, \\ 22761 Hamburg, Germany \\ ${ }^{2}$ Deutsches Elektronen-Synchrotron DESY, Notkestraße 85, 22607 Hamburg, Germany \\ ${ }^{3}$ Christian-Albrechts-Universität zu Kiel, Max-Eyth-Str.1, 24118 Kiel, Germany
}

(Received 21 July 2017; accepted 12 September 2017; published online 29 September 2017)

\begin{abstract}
We use high-resolution rotational spectroscopy to investigate the structural intricacies of the lactone form of mevalonic acid, precursor of the mevalonate pathway. By combining microwave spectroscopy with supersonic expansions and quantum-chemical calculations, we determine the two most stable conformations of the precursor. Complementary micro-solvation studies reveal that aggregation of the first water molecule induces a substantial structural rearrangement comprising a hydroxy rotation and an endocyclic core torsion to create a favourable geometry to accommodate the water molecule. We discuss the conformational aspects of the precursor in isolation and under micro-hydrated conditions. Published by AIP Publishing. https://doi.org/10.1063/1.4997162
\end{abstract}

\section{INTRODUCTION}

Understanding the interconnection between the structure and function of complex molecular systems is paramount in bio-chemical science. Metabolic precursors are a good example of fundamentally important molecules where biological function emerges from particular structural properties. Revealing the structural intricacies of these systems is thus an appealing target for spectroscopists in general. Under investigation here is mevalonolactone (MV), the $\delta$-lactone form of mevalonic acid, precursor of the mevalonate pathway. ${ }^{1,2}$ The carboxylate anion of mevalonic acid, known as mevalonate, is the predominant form in biological environments and is of major pharmaceutical importance. The mevalonate pathway converts mevalonate into molecular systems such as cholesterol, among many others. ${ }^{1}$ Drugs such as the statins (which lower the levels of cholesterol) stop the production of mevalonate by inhibiting HMG-CoA reductase. Mevalonic acid is very soluble in water and in polar organic solvents. At physiological $\mathrm{pH}$, an equilibrium is at play between the acid and its $\delta$-lactone form, which is formed through internal condensation of the terminal alcohol and carboxylic acid functional groups. This delicate balance indicates a mediator role of water in natural aqueous environments.

Isolated micro-hydrated molecules in the gas phase provide the conditions to investigate the structural changes induced by a small number of water molecules without the presence of external influences and as such have become an appealing target for high-resolution spectroscopic studies. ${ }^{3-10}$ High-resolution microwave spectroscopy enables the unambiguous identification of molecular species and of thermal distribution of conformations. The unique rotational constants

a)Electronic mail: melanie.schnell@desy.de of a particular conformation of a specific molecule allow for unambiguous differentiation of species from their rotational spectrum. The implementation of chirped-pulse (CP) excitation schemes in Fourier transform microwave spectroscopy (FTMW) has triggered an emergence of broadband rotational spectroscopy for molecular structure determination. ${ }^{11,12}$ Here we report the study of MV and its complex with water using CP-FTMW spectroscopy. The structure of the global minimum is determined using the spectra of singly substituted isotopologues in natural abundance. The structures of a second conformer and of the most stable water complex are assigned through comparison with quantum-chemical calculations. Isotopic substitution studies are performed to confirm the predicted structures for the hydrated species. Noticeable structural changes are induced on the monomer upon aggregation with water. The observed degree of structural reorientation induced by micro-hydration is unusual and brings about interesting insights regarding water-induced structural changes in bio-chemical aqueous media.

\section{METHODS}

\section{A. Experimental}

The sample $( \pm)$-mevalonolactone was purchased from Sigma Aldrich (97\%) and used without further purification. All measurements reported henceforth were performed using the Hamburg COMPACT spectrometer. A complete description of the experimental setup can be found in Ref. 13 and only a brief explanation is provided here. A supersonic expansion brings the sample into the vacuum chamber by means of a pulsed nozzle (Parker General Valve Series 9) operating at $9 \mathrm{~Hz}$ with a constant flow of neon at stagnation pressures of 2 bars to generate a cold molecular beam. The optimum signal was obtained by heating the sample to $90{ }^{\circ} \mathrm{C}$ to create 
sufficient vapour pressure. To seed the supersonic expansion with water, we create a bypass in the Ne backing pressure line. The flow passes through a reservoir containing water before reaching the nozzle, where the sample is being heated. A $4 \mu \mathrm{s}$ chirp spanning 2-8 GHz is generated with an arbitrary waveform generator (AWG) and amplified in a $300 \mathrm{~W}$ traveling wave tube (TWT) amplifier preceding the broadcast into the vacuum chamber via a horn antenna. The chirp polarises the ensemble of molecules in the beam, and we record the free induction decay (FID) of the macroscopic dipole moment of the molecular ensemble. To improve data collection speed and minimise sample consumption, we employed the "fast-frame" option of the digital oscilloscope. ${ }^{14}$ Eight back-to-back chirps excite each gas pulse, and the subsequent eight FID acquisitions are co-added and averaged. The effective repetition rate of the experiment is thus $72 \mathrm{~Hz}$. The microwave spectrum reported consists of $1.5 \times 10^{6}$ averaged spectra, which translates to $6 \mathrm{~h}$ of measurement time. In the second set of experiments, we used a $4 \mu$ s chirp spanning 8-12 GHz and amplified it in a $50 \mathrm{~W}$ solid state amplifier (Mercury Systems). A total of $4 \times 10^{6}$ averaged spectra were collected during $16 \mathrm{~h}$ of measurement time.

\section{B. Theoretical}

The experiments were supported by quantum-chemical calculations using Gaussian09. ${ }^{15}$ From a previous study on the potential energy surface of MV, we identify the lowest energy conformers having the methyl group in the equatorial position. ${ }^{16}$ Ring puckering generates another series of stable conformers but at higher energies. These species will not be considered in this study. A relaxed potential energy scan was obtained with stepwise progressions of the coordinate associated with the rotation of the hydroxy group. The potential energy scan is depicted in Fig. 1. The two minima were further optimised using ab initio and density functional theory methods. To also account for dispersion, the Minessota functionals M06- $2 \mathrm{X}^{17}$, the hybrid Becke 3 Parameter B3LYP including Grimme's dispersion correction ${ }^{18}$ with Becke-Johnson damping, ${ }^{19}$ and Møller-Plesset second-order perturbation theory (MP2) were employed. For all calculations, the aug-cc-pVTZ basis set was used, except for the MP2 calculation for the water cluster, in which we used the $6-311++\mathrm{G}^{* *}$ basis set due to high computational cost. Frequency calculations were

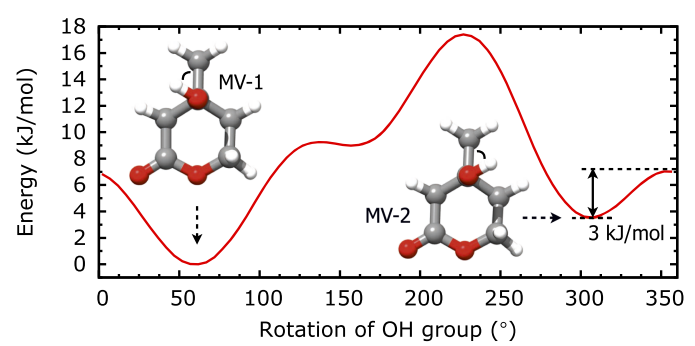

FIG. 1. Relaxed potential energy scan of the coordinate comprising rotation of the hydroxy group of MV. The calculation was performed using density functional theory at the B3LYP-D3(BJ)/6-311++G** level of theory. MV-1 and $\mathrm{MV}-2$ are the global and local minima, respectively, captured in the scan. Zero-point corrected energies were calculated at the B3LYP-D3(BJ)/aug-cc$\mathrm{pVTZ}$ level of theory for both conformers yielding $\Delta E_{\mathrm{MV}-1}=0$ and $\Delta E_{\mathrm{MV}-2}$ $=2.1 \mathrm{~kJ} / \mathrm{mol}$. performed for all reported molecular species and their zeropoint corrected energies determined.

Starting structures for the complex of MV with water were initially obtained using the program ABCluster. ${ }^{20}$ Using this algorithm, the conformations of monomeric species are constrained preventing any structural reorientation due to intermolecular interactions to occur. All the predicted MV-water complexes were then further optimised using the B3LYPD3(BJ)/def2-pVTZ level of theory. Of the fifteen computed complexes, four converged to the lowest energy form, which we will discuss later in this paper. The remaining complexes are predicted with energies higher than $6 \mathrm{~kJ} / \mathrm{mol}$ and thus were disregarded in our study.

\section{RESULTS AND DISCUSSION}

A segment of the broadband rotational spectrum of MV is shown in Fig. 2. The upper and lower panels show the same frequency range but are scaled in intensity to highlight the weaker transitions. In the upper panel, we show the experimental spectrum (black trace) and a simulation based on the fitted spectroscopic parameters (red trace) reported in Table I. In the $2-8 \mathrm{GHz}$ frequency range, we cover mainly a-type transitions, denoted by the rotational quantum numbers $J, K_{a}$, and $K_{c}$, with $J$ being the rotational angular momentum quantum number and $K_{a}$ and $K_{c}$ being the projections of $J$ onto the principal axes at the prolate and oblate symmetric top limits, respectively. The depicted pattern $(J+1) \leftarrow J=3 \leftarrow 2$ represents the most intense of three groups of a-type transitions between the $J$ levels captured in this range. In the second measurement, we expanded the range until $12 \mathrm{GHz}$, thus capturing the $(J+1) \leftarrow J=4 \leftarrow 3$ group of rotational transitions.

A total of 60 rotational transitions were assigned to the predominant species in the spectrum, which we confidently assign to conformation MV-1 (see Table I) based on direct comparison with quantum-chemical calculations. Experimental rotational constants were determined through a recurrent fit using the A-reduced rigid rotor Hamiltonian as

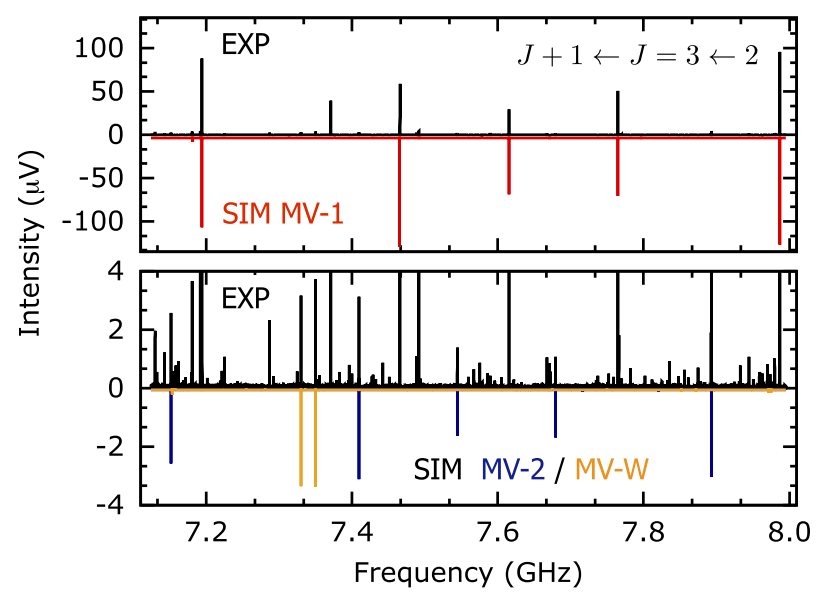

FIG. 2. Segment of the broadband rotational spectrum of MV from 7 to $8 \mathrm{GHz}$. The upper traces (in both upper and lower panels) show the experimental spectra using neon as a carrier gas. The lower traces represent simulations obtained from the fitted spectroscopic parameters reported in Table I. The lower panel is in the same frequency range as the upper panel but scaled in intensity to highlight the weaker transitions. Most of the remaining weaker transitions visible in the experimental spectrum belong to isotopologues of MV-1. 
TABLE I. Experimentally determined parameters for the two conformers of $\mathrm{MV}$ and the complex of MV with water identified in the microwave spectrum: rotational constants (A, B, C in MHz) and quartic centrifugal distortion constants (in $\mathrm{kHz}$ ); type of rotational transitions observed (a-type, b-type, c-type) with y being observed and $\mathrm{n}$ being not observed; predicted dipole moments; number of lines included into the fit; standard error of the fit $(\mathrm{kHz})$. The experimental frequency accuracy is $25 \mathrm{kHz}$.

\begin{tabular}{|c|c|c|c|c|}
\hline \multicolumn{5}{|c|}{ MV-1 } \\
\hline & Expt. & M062X & $\mathrm{MP} 2^{\mathrm{a}}$ & B3LYP-D3(BJ) \\
\hline $\mathrm{A}(\mathrm{MHz})$ & $2627.13492(55)$ & 2649.967 & 2640.358 & 2628.3793 \\
\hline $\mathrm{B}(\mathrm{MHz})$ & $1401.92970(51)$ & 1417.179 & 1415.132 & 1400.381 \\
\hline $\mathrm{C}(\mathrm{MHz})$ & $1136.44913(53)$ & 1147.081 & 1145.913 & 1134.280 \\
\hline $\mathrm{D}_{J}(\mathrm{kHz})$ & $0.125(15)$ & $\ldots$ & $\ldots$ & $\ldots$ \\
\hline$\left|\mu_{a}\right|(\mathrm{D})$ & $\mathrm{y}$ & 4.8 & 4.7 & 4.7 \\
\hline$\left|\mu_{b}\right|$ (D) & $\mathrm{y}$ & 0.4 & 0.5 & 0.5 \\
\hline$\left|\mu_{c}\right|(\mathrm{D})$ & $\mathrm{y}$ & 1.2 & 0.8 & 1.2 \\
\hline $\mathrm{N}$ & 60 & $\ldots$ & $\ldots$ & $\ldots$ \\
\hline$\sigma(\mathrm{kHz})$ & 7.4 & $\ldots$ & $\ldots$ & $\ldots$ \\
\hline \multicolumn{5}{|c|}{ MV-2 } \\
\hline & Expt. & M062X & $\mathrm{MP}^{\mathrm{a}}$ & B3LYP-D3(BJ) $)^{\mathrm{a}}$ \\
\hline $\mathrm{A}(\mathrm{MHz})$ & 2577.191(33) & 2654.528 & 2643.338 & 2632.353 \\
\hline B (MHz) & $1381.62419(95)$ & 1407.764 & 1406.082 & 1391.720 \\
\hline $\mathrm{C}(\mathrm{MHz})$ & $1133.18674(84)$ & 1141.947 & 1140.901 & 1129.685 \\
\hline$\left|\mu_{a}\right|(\mathrm{D})$ & $\mathrm{y}$ & 5.6 & 5.4 & 5.6 \\
\hline$\left|\mu_{b}\right|$ (D) & $\mathrm{n}$ & 2.2 & 2.5 & 2.2 \\
\hline$\left|\mu_{c}\right|$ (D) & $\mathrm{n}$ & 1.3 & 1.2 & 1.4 \\
\hline $\mathrm{N}$ & 14 & $\ldots$ & $\ldots$ & $\ldots$ \\
\hline$\sigma(\mathrm{kHz})$ & 7.6 & $\ldots$ & & \\
\hline \multicolumn{5}{|c|}{ MV-W } \\
\hline & Expt. & M062X & $\mathrm{MP} 2^{\mathrm{b}}$ & B3LYP-D3(BJ) ${ }^{\mathrm{a}}$ \\
\hline $\mathrm{A}(\mathrm{MHz})$ & $1485.4274(28)$ & 1508.921 & 1471.360 & 1498.953 \\
\hline B (MHz) & $1224.2147(12)$ & 1263.223 & 1263.358 & 1237.435 \\
\hline $\mathrm{C}(\mathrm{MHz})$ & $857.32848(79)$ & 870.464 & 859.006 & 864.787 \\
\hline $\mathrm{D}_{J}(\mathrm{kHz})$ & $0.193(11)$ & $\ldots$ & $\ldots$ & $\ldots$ \\
\hline $\mathrm{d}_{J}(\mathrm{kHz})$ & $0.0518(94)$ & $\ldots$ & $\ldots$ & $\ldots$ \\
\hline$\left|\mu_{a}\right|(\mathrm{D})$ & $\mathrm{y}$ & 2.2 & 1.7 & 2.3 \\
\hline$\left|\mu_{b}\right|$ (D) & $\mathrm{n}$ & 0.8 & 1.3 & 0.3 \\
\hline$\left|\mu_{c}\right|$ (D) & $\mathrm{n}$ & 0.4 & 0.4 & 0.2 \\
\hline $\mathrm{N}$ & 24 & $\ldots$ & $\ldots$ & $\ldots$ \\
\hline$\sigma / \mathrm{kHz}$ & 5.6 & $\ldots$ & $\ldots$ & $\ldots$ \\
\hline
\end{tabular}

a aug-cc-pVTZ basis set.

b $6-311++\mathrm{G}^{* *}$ basis set.

implemented in PGOPHER. ${ }^{21}$ The quartic centrifugal distortion constant $\mathrm{D}_{J}$ was also determined. A summary of the fitted spectroscopic parameters is shown in Table I. The B3LYP$\mathrm{D} 3(\mathrm{BJ}) /$ aug-cc-pVTZ level of theory is the most accurate in predicting the rotational constants for MV-1 with less than $0.2 \%$ deviation between experiment and theory.

A number of rotational transitions for MV-1 overcome a signal-to-noise ratio of 600:1, allowing the observation of satellite transitions arising from the singly substituted ${ }^{13} \mathrm{C}$ isotopologues in natural abundance $(1.1 \%)$. With these conditions, we were able to observe and fit all the spectra from the isotopologues and use their rotational constants to employ the substitution, $\mathrm{r}_{s}$, method to derive the positions of all carbon atoms in the principal axis frame using the Kraitchman equations. ${ }^{22}$ The atomic coordinates can be extracted from the changes in the moments of inertia upon isotopic substitution. In addition, we employed the $r_{0}$ method ${ }^{23}$ where the effective structure of the ground state is here partially determined by a least squares fit using the complete set of rotational parameters.

A comparison between the parameters for the $\mathrm{r}_{s}, \mathrm{r}_{0}$, and calculated structures for MV-1 is shown in Fig. 3. An overlay of the $r_{s}$ and calculated structures is depicted. Relevant bond lengths and endocyclic dihedrals for MV-1 are given in Table II. Additional data, including the results from the Kraitchman calculation, are reported in the supplementary material. We note that the agreement between experiment and theory is good, and all bond lengths and endocyclic torsions are well predicted by the employed theoretical methods. We find that the $r_{0}$ structure is particularly well reproduced by the quantum-chemical methods.

In Fig. 2 (lower panel), we show a magnified segment of the broadband spectrum where we assign rotational transitions for a second conformer. By comparison with quantum chemistry methods, we can confidently assign our fitted rotational constants to conformer MV-2, which relates to MV-1 by a rotation of the $\mathrm{OH}$ group as indicated in Fig. 1. The fitted spectroscopic parameters can be found in Table I. Only fourteen rotational transitions could be assigned for this conformer since the signal level is considerably weaker compared with that of MV-1. That is partially explained by the energy of MV-2 with respect to the global minimum and the low barrier separating MV-1 and MV-2 (see Fig. 1), which likely implies that conformational cooling occurs during the supersonic expansion. Also, the rather large rotational constants of MV are not optimally suited for the frequency range of our instrument since we only capture a tale of the rotational profile at $2 \mathrm{~K}$. The predicted permanent dipole moment components of MV-2 show a large a-type contribution in prejudice of $b$ and $c$ components much like MV-1. As a consequence, only a-type rotational transitions could be assigned for $\mathrm{MV}-2$, and since our frequency range only covers four $J$ levels

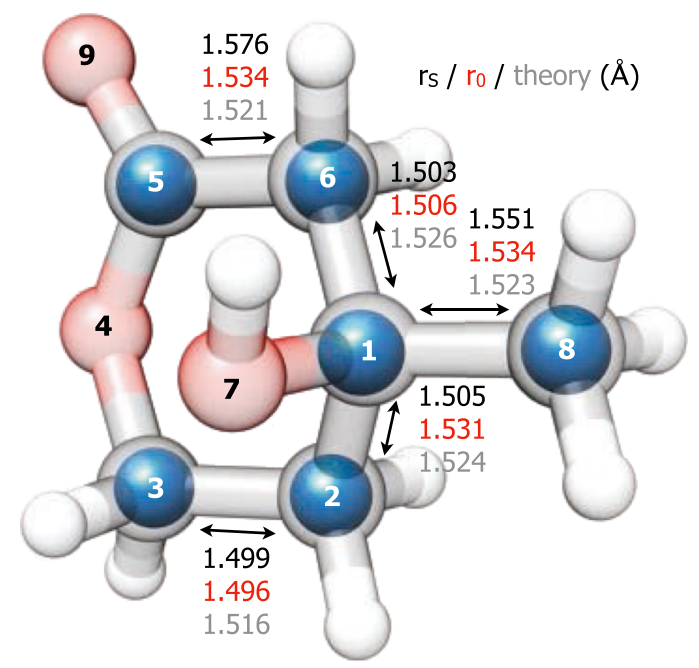

FIG. 3. Overlay of the $\mathrm{r}_{s}$ and predicted [B3LYP-D3(BJ)/aug-cc-pVTZ] structures of MV-1. The solid spheres represent the experimentally $\left(\mathrm{r}_{s}\right)$ determined carbon atom positions. Relevant bond lengths are depicted for the $\mathrm{r}_{s}, \mathrm{r}_{0}$, and calculated structures of MV. Endocyclic torsions are given in Table II. 
TABLE II. Experimental $\left(r_{s}\right.$ and $\left.r_{0}\right)$ and calculated bond lengths and endocyclic dihedral angles for MV-1. The numbering given for the bond lengths and dihedral angles refers to the atom numbering of Fig. 3.

\begin{tabular}{lccccccc}
\hline \hline$(\AA)$ & $\mathrm{r}_{s}$ & Std. dev. & $\mathrm{r}_{0}$ & Std. dev. & $\mathrm{Calc}^{\mathrm{a}}$ & $\Delta\left(\mathrm{r}_{s}\right)^{\mathrm{b}}(\%)$ & $\Delta\left(\mathrm{r}_{0}\right)^{\mathrm{b}}(\%)$ \\
\hline $\mathrm{r}(1-2)$ & 1.505 & 0.022 & 1.531 & 0.003 & 1.524 & 1.24 & 0.45 \\
$\mathrm{r}(2-3)$ & 1.499 & 0.087 & 1.496 & 0.005 & 1.514 & 0.99 & 1.18 \\
$\mathrm{r}(5-6)$ & 1.576 & 0.011 & 1.534 & 0.006 & 1.518 & 3.80 & 1.05 \\
$\mathrm{r}(6-1)$ & 1.503 & 0.017 & 1.506 & 0.006 & 1.528 & 1.63 & 1.43 \\
$\mathrm{r}(1-8)$ & 1.551 & 0.021 & 1.534 & 0.003 & 1.523 & 1.83 & 0.72 \\
\hline$\left.{ }^{\circ}\right)$ & & & & & & & \\
\hline$\phi(5-6-1-2)$ & +49.665 & 3.434 & +46.321 & 0.142 & +45.830 & 8.36 & 1.07 \\
$\phi(6-1-2-3)$ & -60.619 & 7.722 & -59.079 & 0.214 & -58.260 & 4.04 & 1.40 \\
\hline \hline
\end{tabular}

${ }^{\mathrm{a} B 3 L Y P-D 3(B J) / a u g-c c-p V T Z ~ b a s i s ~ s e t . ~}$

${ }^{\mathrm{b}}$ Percentual differences $\Delta\left(\mathrm{r}_{s}\right)$ and $\Delta\left(\mathrm{r}_{0}\right)$ are calculated as $\frac{c a l c-r_{s}}{c a l c} \times 100$ and $\frac{c a l c-r_{0}}{\text { calc }} \times 100$, respectively.

for this molecule, the number of transitions is limited. Nevertheless, the rotational constants could be determined and compared with quantum-chemical calculations for a confident assignment. We note that the experimental rotational constant $\mathrm{A}$ is slightly overestimated by all three quantum chemistry methods.

In a different set of experiments, we co-expanded our sample with water to generate water clusters and thus investigate MV under hydrating conditions. The lower panel of Fig. 2 depicts a magnified segment of the microwave spectrum where rotational lines arising from hydrated species can be identified. A total of 24 rotational transitions were assigned, and the rotational constants were determined using the same methodology previously described. Again, the B3LYP-D3(BJ)/augcc-pVTZ level of theory is the one that better predicts our observations. The spectroscopic parameters are given in Table I. All rotational constants are well determined. Quartic centrifugal distortion constants $D_{J}$ and $d_{J}$ were also included in the fit. To further confirm the geometry of the MV-water cluster, and in particular to reveal the exact position of the bound water molecule, we performed an additional experiment where we seeded the molecular jet with an isotopically enriched sample of water with ca. $50 \% \mathrm{H}_{2}{ }^{18} \mathrm{O}$ water $(500 \mathrm{k}$ averages, $2 \mathrm{~h}$ of measurement time). A total of 16 rotational transitions could be assigned in the $6-12 \mathrm{GHz}$ frequency range for the $\mathrm{MV}-\mathrm{H}_{2}{ }^{18} \mathrm{O}$ species. The rotational constants and rotational frequencies are given in the supplementary material. Using the $\mathrm{r}_{s}$ method, we experimentally determined the position of the water oxygen atom in the MV-W cluster, therefore verifying the $a b$ initio structure and confidently assigning the geometry of the cluster. An overlay of the structures for MV-1 and MV-W is shown in Fig. 4.

A remarkable geometrical feature of the complex of MV with water is that, besides a noticeable endocyclic core structural change, the hydroxy group undergoes a clear reorientation $\left(60^{\circ} \rightarrow 92^{\circ}\right)$ with respect to MV-1 (see Fig. 4). Although not without precedent, ${ }^{24-29}$ such large reorientation is unusual and brings about interesting insights regarding water induced structural changes. While most of the endocyclic torsions are not dramatically affected upon aggregation of water, the pseudo-axial $\mathrm{OH}$ group is relatively free to rotate to mediate the complexation as a hydrogen-bond donor. The carbonyl group positioned in the equatorial position with respect to the hydroxy group functions as the expected hydrogen-bond acceptor. The intramolecular link between these two moieties, that is the hydroxy and carbonyl groups, is efficiently achieved via the connection created by the water molecule.

Our initial theoretical investigations of complex formation between MV and water using ABCluster led to a series of candidate complex geometries that we further refined using DFT geometry optimisation methods. Out of the 15 starting geometries, four structures converged to the geometry assigned to the observed MV-W species. All other candidates were discarded because of their higher relative energies. In Fig. 5, we show the electronic energy (lower panel), dihedral angle $\tau$ reflecting the relative orientation of the $\mathrm{OH}$ group with respect to the methyl group (middle panel), and endocyclic torsion $\alpha$ (upper panel) during the geometry optimisation of all four complexes at the B3LYP-D3(BJ)/def2-TZVP level of theory. The energy and starting coordinates are marked for both MV-1 and MV-W to show their evolution during the optimisation procedure. It is readily evident that in all four cases, the energy is rapidly minimised following a trend towards the coordinates $\alpha$ and $\tau$ of MV-W. Moreover, we note that the starting position of the water molecule differs for all four species, in particular for the starting complex indicated by the green trace, where we find the water molecule in an equatorial position (see supplementary material). A clear stabilisation is achieved via the cluster formation for all predicted trajectories.

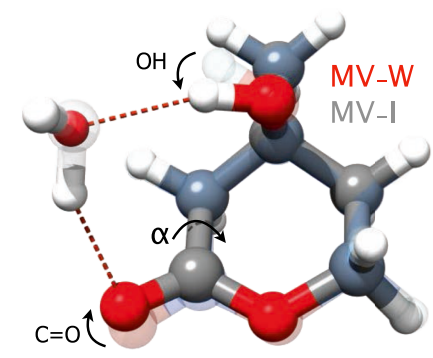

view 1

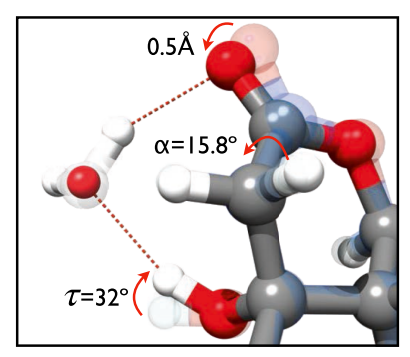

view 2
FIG. 4. Overlays of calculated structures [B3LYP-D3(BJ)/aug-cc-pVTZ] for MV-1 (transparent) and MV-W (full colour) depicting the degree of reorientation of the hydroxy and carbonyl groups. The selected angle $\alpha$ indicates the endocyclic dihedral $\phi(4-5-6-1)$, which changes the most upon complexation with water (see Table III). The red sphere overlaid with the water molecule depicts the experimentally determined position of the water oxygen atom from the $\mathrm{H}_{2}{ }^{18} \mathrm{O}$ measurement. 


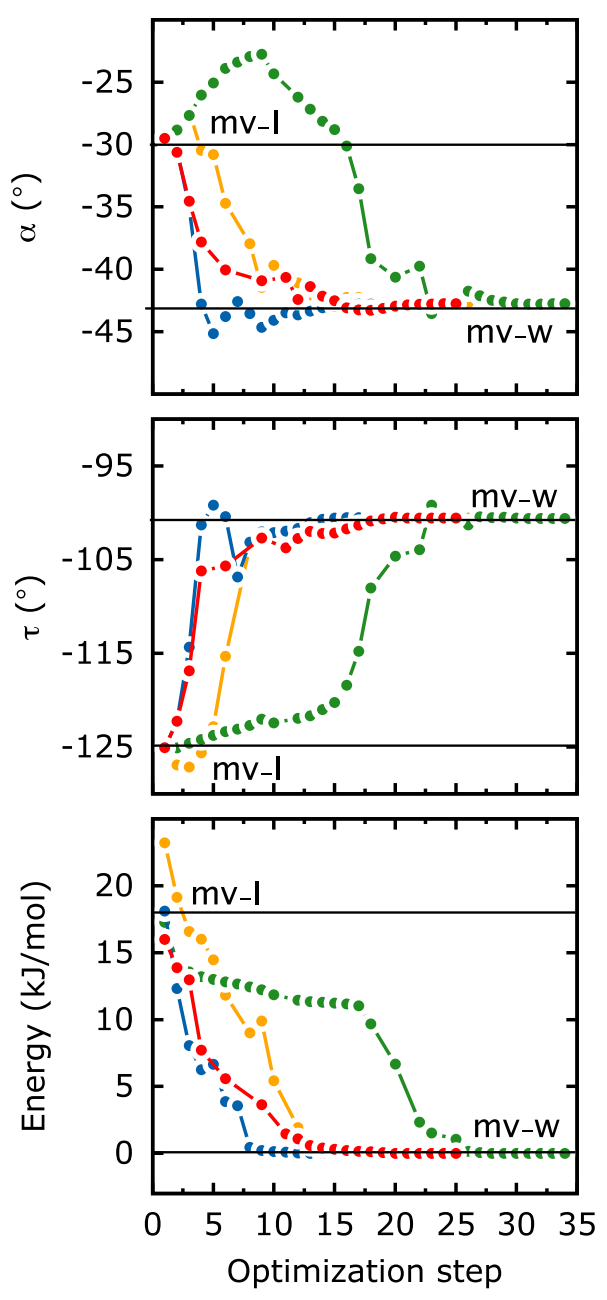

FIG. 5. Calculated electronic energy (lower panel), dihedral angle $\tau$ (middle panel), and endocyclic torsion $\alpha$ (upper panel) during the geometry optimisation of four MV-W complexes at the B3LYP-D3(BJ)/def2-TZVP level of theory (a different basis set was employed here to reduce computational cost associated with the search for MV-water clusters). The structures of the four relevant species and of the observed MV-W complex are given in the supplementary material. The horizontal traces (in black) indicate the coordinates of the stable MV-1 and MV-W species at the same level of theory, as a guide to the eye.

In Table III, we report the endocyclic torsions for all observed MV species and those of $\delta$-valerolactone (same endocyclic core structure without the $\mathrm{OH}$ and $\mathrm{CH}_{3}$ groups) for a detailed comparison and to evaluate the degree of structural changes induced by hydration. In fact, while the endocyclic core structure is rather similar for MV-1, MV-2 and $\delta$-v, noticeable changes occur for MV-W. In particular, we note the most

TABLE III. Calculated endocyclic dihedral angles for MV-1, MV-2, MV-W, and $\delta$-valerolactone $(\delta$-v).

\begin{tabular}{lccccc}
\hline \hline$\left.{ }^{\circ}\right)$ & $\mathrm{MV}^{\mathrm{a}} 1^{\mathrm{a}}$ & $\mathrm{MV}-\mathrm{W}^{\mathrm{a}}$ & $\mathrm{MV}^{\mathrm{a}}$ & $\delta^{\mathrm{a}}$-v. $^{\mathrm{a}}$ & $\mathrm{MV}(1-\mathrm{W})^{\mathrm{a}}$ \\
\hline$\phi(1-2-3-4)$ & +52.74 & +46.41 & +54.60 & +53.56 & +6.33 \\
$\phi(2-3-4-5)$ & -33.00 & -30.96 & -34.49 & -32.11 & -2.03 \\
$\phi(3-4-5-6)$ & +19.54 & +28.87 & +18.18 & +15.84 & -9.32 \\
$\phi(4-5-6-1)$ & -26.81 & -42.68 & -23.11 & -22.53 & +15.86 \\
$\phi(5-6-1-2)$ & +45.83 & +56.80 & +42.63 & +44.45 & -10.97 \\
$\phi(6-1-2-3)$ & -58.26 & -59.06 & -57.74 & -59.20 & +0.80 \\
\hline
\end{tabular}

aB3LYP-D3(BJ)/aug-cc-pVTZ.
TABLE IV. Energy decomposition (kJ/mol) obtained from a SAPT(0)/juncc-pVDZ calculation on the MV-W complex and on the water dimer for comparison. $\Delta \mathrm{E}_{\text {elec }}$ is the electrostatic energy; $\Delta \mathrm{E}_{\text {exch }}$ represents the repulsion due to exchange; $\Delta \mathrm{E}_{\text {ind }}$ induction energy accounting charge transfer interactions; $\Delta \mathrm{E}_{\mathrm{disp}}$ is the energy contribution from dispersion interactions.

\begin{tabular}{lccrrr}
\hline \hline & $\Delta \mathrm{E}_{\text {elec }}$ & $\Delta \mathrm{E}_{\text {exch }}$ & $\Delta \mathrm{E}_{\text {ind }}$ & $\Delta \mathrm{E}_{\text {disp }}$ & $\Delta \mathrm{E}_{\text {tot }}$ \\
\hline $\mathrm{MV}-\mathrm{W}$ & -76.6 & 65.5 & -21.7 & -17.2 & -50.0 \\
$\left(\mathrm{H}_{2} \mathrm{O}\right)_{2}$ & -36.2 & 28.4 & -8.5 & -5.2 & -21.6 \\
\hline \hline
\end{tabular}

relevant torsional change of about $15^{\circ}$ corresponding to the dihedral $\alpha$ as depicted is Fig. 4. An overlay of the structures of $\mathrm{MV}-1$ and MV-W is given in Fig. 4 to provide the reader with an overview of the degree of structural rearrangement occurring for the hydrated species and compared with that of the global minimum structure MV-1. It seems that a cooperative structural rearrangement occurs in order to more efficiently bind the guest water molecule. This cooperation includes the reorientation of the $\mathrm{OH}$ group by $32^{\circ}$ and the torsion of the dihedral coordinate $\alpha$ comprising atoms 4, 5, 6 and 1 (see Fig. 3). This torsion effectively bends the carbonyl group from an equatorial orientation towards an axial one.

To further elucidate the forces at play in this particular water binding topology, we performed zeroth-order symmetry adapted perturbation theory (SAPT) ${ }^{30}$ calculations to decompose the energetic contributions arising from the intermolecular binding forces in MV-W. A SAPT(0)/jun-cc-pVDZ ${ }^{31}$ calculation was performed for the complex using the Psi 4 electronic structure package. ${ }^{32}$ The results of this calculation are presented in Table IV. As expected, the predominant contribution at play is of electrostatic nature, which arises mainly from the dual donor-acceptor $\mathrm{O}-\mathrm{H} \cdots \mathrm{O}$ topology created by the water molecule interconnecting the two MV moieties. These contributions are of the same order of magnitude as in the water dimer. Dispersion interactions play a secondary role in this complex, similarly to the water dimer.

\section{CONCLUSIONS}

In summary, we reported on the conformational preferences of MV in isolation and in a microsolvated environment using high-resolution rotational spectroscopy. Besides the elucidation of the monomeric ground state structure and of a second conformation at higher energy, we investigated the structural changes induced in the monomer under controlled hydration conditions. For that, we generated clusters of mevalonolactone and water in a supersonic jet. We observe a molecular complex of MV with one water molecule and find that substantial structural changes occur to MV to efficiently host the bound water molecule. These changes comprise reorientation of the hydroxy group and torsion of the endocyclic core structure. Our observations thus provide a new perspective on the structure of MV in hydrated media: previous studies with MV using Raman optical activity in neat liquid and aqueous solutions indicate no apparent structural change due to the presence of water. ${ }^{16}$ Under micro-solvated conditions, however, we show that water aggregation clearly mediates the conformational reshaping of MV. This observation could shed light onto the balance achieved between the acid and lactone 
forms of MV in physiological environments. Moreover, this result is particularly interesting if one considers that under appropriate hydration conditions, the conformation of certain drugs such as statins (which contain the MV moiety) can be altered and consequently its biological activity influenced by hydration.

\section{SUPPLEMENTARY MATERIAL}

See supplementary material for rotational constants of all isotopic species, Kraitchman calculations, and line lists with fitted rotational transitions.

\section{ACKNOWLEDGMENTS}

This work has been supported by the excellence cluster "the Hamburg Centre for Ultrafast Imaging-Structure, Dynamics and Control of Matter at the Atomic Scale" of the Deutsche Forschungsgemeinschaft. S.R.D. acknowledges a Postdoctoral Research Fellowship from the Alexander von Humboldt Foundation. We acknowledge the use of the GWDG computer cluster.

${ }^{1}$ I. Buhaescu and H. Izzedine, "Mevalonate pathway: A review of clinical and therapeutical implications," Clin. Biochem. 40, 575-584 (2007).

${ }^{2}$ P. J. Mullen, R. Yu, J. Longo, M. C. Archer, and L. Z. Penn, "The interplay between cell signalling and the mevalonate pathway in cancer," Nat. Rev. Cancer 16, 718-731 (2016).

${ }^{3}$ W. Caminati, P. Moreschini, and P. G. Favero, "The hydrogen bond between water and aromatic bases of biological interest: Rotational spectrum of pyridazine-water," J. Phys. Chem. A 102, 8097-8100 (1998).

${ }^{4}$ L. B. Favero and W. Caminati, "Hydrated complexes of atmospheric interest: Rotational spectrum of diacetyl-water," J. Phys. Chem. A 113, 1430814311 (2009).

${ }^{5}$ C. Pérez, J. L. Neill, M. T. Muckle, D. P. Zaleski, I. Peña, J. C. López, J. L. Alonso, and B. H. Pate, "Water-water and water-solute interactions in microsolvated organic complexes," Angew. Chem., Int. Ed. 54, 979-982 (2015).

${ }^{6}$ E. G. Schnitzler, C. Badran, and W. Jäger, "Contrasting effects of water on the barriers to decarboxylation of two oxalic acid monohydrates: A combined rotational spectroscopic and ab initio study," J. Phys. Chem. Lett. 7, 1143-1147 (2016).

${ }^{7}$ C. Pérez, A. Krin, A. L. Steber, J. C. López, Z. Kisiel, and M. Schnell, "Wetting camphor: Multi-isotopic substitution identifies the complementary roles of hydrogen bonding and dispersive forces," J. Phys. Chem. Lett. 7, 154-160 (2016).

${ }^{8}$ S. Blanco, P. Pinacho, and J. C. López, "Hydrogen-bond cooperativity in formamide2-water: A model for water-mediated interactions," Angew. Chem., Int. Ed. 55, 9331-9335 (2016).

${ }^{9}$ S. R. Domingos, C. Pérez, and M. Schnell, "Communication: Structural locking mediated by a water wire: A high-resolution rotational spectroscopy study on hydrated forms of a chiral biphenyl derivative," J. Chem. Phys. 145, 161103 (2016)

${ }^{10}$ E. G. Schnitzler, N. A. Seifert, S. Ghosh, J. Thomas, Y. Xu, and W. Jäger, "Hydration of the simplest $\alpha$-keto acid: A rotational spectroscopic and $a b$ initio study of the pyruvic acid-water complex," Phys. Chem. Chem. Phys. 19, 4440-4446 (2017)

${ }^{11}$ G. G. Brown, B. C. Dian, K. O. Douglass, S. M. Geyer, S. T. Shipman, and B. H. Pate, "A broadband Fourier transform microwave spectrometer based on chirped pulse excitation," Rev. Sci. Instrum. 79, 053103 (2008).

${ }^{12}$ B. G. Park and R. W. Field, "Perspective: The first ten years of broadband chirped pulse Fourier transform microwave spectroscopy," J. Chem. Phys. 144, 200901 (2016).

${ }^{13}$ D. Schmitz, V. Alvin Shubert, T. Betz, and M. Schnell, "Multi-resonance effects within a single chirp in broadband rotational spectroscopy: The rapid adiabatic passage regime for benzonitrile," J. Mol. Spectrosc. 280, 77-84 (2012).

${ }^{14}$ C. Pérez, S. Lobsiger, N. A. Seifert, D. P. Zaleski, B. Temelso, G. C. Shields, Z. Kisiel, and B. H. Pate, "Broadband Fourier transform rotational spectroscopy for structure determination: The water heptamer," Chem. Phys. Lett. 571, 1-15 (2013).

${ }^{15}$ M. Frisch, G. Trucks, H. Schlegel, G. Scuseria, M. Robb, J. Cheeseman, G. Scalmani, V. Barone, B. Mennucci, G. Petersson, H. Nakatsuji, M. Caricato, X. Li, H. Hratchian, A. Izmaylov, J. Bloino, G. Zheng, J. Sonnenberg, M. Hada, M. Ehara, K. Toyota, R. Fukuda, J. Hasegawa, M. Ishida, T. Nakajima, Y. Honda, O. Kitao, H. Nakai, T. Vreven, J. Montgomery, J. Peralta, F. Ogliaro, M. Bearpark, J. Heyd, E. Brothers, K. Kudin, V. Staroverov, R. Kobayashi, J. Normand, K. Raghavachari, A. Rendell, J. Burant, S. Iyengar, J. Tomasi, M. Cossi, N. Rega, J. Millam, M. Klene, J. Knox, J. Cross, V. Bakken, C. Adamo, J. Jaramillo, R. Gomperts, R. Stratmann, O. Yazyev, A. Austin, R. Cammi, C. Pomelli, J. Ochterski, R. Martin, K. Morokuma, V. Zakrzewski, G. Voth, P. Salvador, J. Dannenberg, S. Dapprich, A. Daniels, O. Farkas, J. Foresman, J. Ortiz, J. Cioslowski, and D. Fox, Gaussian 09, Revision 2, Gaussian, Inc., 2009.

${ }^{16}$ K. Chruszcz-Lipska, A. Jaworska, E. Szczurek, and M. Baranska, “(-)-RMevalonolactone studied by ROA and SERS spectroscopy," Chirality 26, 453-461 (2014).

${ }^{17}$ Y. Zhao and D. G. Truhlar, "The M06 suite of density functionals for main group thermochemistry, thermochemical kinetics, noncovalent interactions, excited states, and transition elements: Two new functionals and systematic testing of four M06-class functionals and 12 other functionals," Theor. Chem. Acc. 120, 215-241 (2008).

${ }^{18}$ S. Grimme, J. Antony, S. Ehrlich, and H. Krieg, "A consistent and accurate $a b$ initio parametrization of density functional dispersion correction (DFTD) for the 94 elements H-Pu," J. Chem. Phys. 132, 154104 (2010).

${ }^{19} \mathrm{~S}$. Grimme, S. Ehrlich, and L. Goerigk, "Effect of the damping function in dispersion corrected density functional theory," J. Comput. Chem. 32, 1456-1465 (2011).

${ }^{20}$ J. Zhang and M. Dolg, "ABCluster: The artificial bee colony algorithm for cluster global optimization,” Phys. Chem. Chem. Phys. 17, 24173-24181 (2015).

${ }^{21}$ C. M. Western, "PGOPHER: A program for simulating rotational, vibrational and electronic spectra," J. Quant. Spectrosc. Radiat. Transfer 186, 221 (2017).

${ }^{22}$ J. Kraitchman, "Determination of molecular structure from microwave spectroscopic data," Am. J. Phys. 21, 17-24 (1953).

${ }^{23} \mathrm{Z}$. Kisiel, "Least-squares mass-dependence molecular structures for selected weakly bound intermolecular clusters," J. Mol. Spectrosc. 218, 58-67 (2003).

${ }^{24}$ M. J. Tubergen, C. R. Torok, and R. J. Lavrich, "Effect of solvent on molecular conformation: Microwave spectra and structures of 2-aminoethanol van der Waals complexes," J. Chem. Phys. 119, 8397-8403 (2003).

${ }^{25}$ B. M. Giuliano and W. Caminati, "Isotopomeric conformational change in anisole-water," Angew. Chem., Int. Ed. 44, 603-606 (2005).

${ }^{26}$ H. Fricke, K. Schwing, A. Gerlach, C. Unterberg, and M. Gerhards, "Investigations of the water clusters of the protected amino acid Ac-Phe-OMe by applying IR/UV double resonance spectroscopy: Microsolvation of the backbone," Phys. Chem. Chem. Phys. 12, 3511-3521 (2010).

${ }^{27}$ A. R. Conrad, N. H. Teumelsan, P. E. Wang, and M. J. Tubergen, "A spectroscopic and computational investigation of the conformational structural changes induced by hydrogen bonding networks in the glycidol-water complex," J. Phys. Chem. A 114, 336-342 (2010).

${ }^{28}$ H. Zhu, M. Blom, I. Compagnon, A. M. Rijs, S. Roy, G. von Helden, and B. Schmidt, "Conformations and vibrational spectra of a model tripeptide: Change of secondary structure upon micro-solvation," Phys. Chem. Chem. Phys. 12, 3415-3425 (2010).

${ }^{29}$ J. Thomas, O. Sukhorukov, W. Jäger, and Y. Xu, "Direct spectroscopic detection of the orientation of free $\mathrm{OH}$ groups in methyl lactate-(water) $)_{1,2}$ clusters: Hydration of a chiral hydroxy ester," Angew. Chem., Int. Ed. 53, 1156-1159 (2014).

${ }^{30}$ B. Jeziorski, R. Moszynski, and K. Szalewicz, "Perturbation theory approach to intermolecular potential energy surfaces of van der Waals complexes," Chem. Rev. 94, 1887-1930 (1994).

${ }^{31}$ T. M. Parker, L. A. Burns, R. M. Parrish, A. G. Ryno, and C. D. Sherrill, "Levels of symmetry adapted perturbation theory (SAPT). I. Efficiency and performance for interaction energies," J. Chem. Phys. 140, 094106 (2014)

${ }^{32}$ J. M. Turney, A. C. Simmonett, R. M. Parrish, E. G. Hohenstein, F. A. Evangelista, J. T. Fermann, B. J. Mintz, L. A. Burns, J. J. Wilke, M. L. Abrams, N. J. Russ, M. L. Leininger, C. L. Janssen, E. T. Seidl, W. D. Allen, H. F. Schaefer, R. A. King, E. F. Valeev, C. D. Sherrill, and T. D. Crawford, "Psi4: An open-source ab initio electronic structure program," Wiley Interdiscip. Rev.: Comput. Mol. Sci. 2, 556-565 (2012). 